\title{
A Study on Clinical Screening of Neonatal Congenital Heart Disease in Jinjiang City
}

\author{
Yu-Lin Zhang' \\ Hai-Tao Bai ${ }^{2}$ \\ 'Department of Pediatric, Jinjiang \\ Municipal Hospital, Jinjiang, People's \\ Republic of China; ${ }^{2}$ Department of \\ Pediatrics, First Affiliated Hospital of \\ Xiamen University, Xiamen, People's \\ Republic of China
}

\begin{abstract}
Objective: This study explored the feasibility of congenital heart disease (CHD) screening by combining a percutaneous oxygen saturation (POX) test with cardiac auscultation method in neonates.

Methods: POX tests and cardiac auscultation were used concurrently to screen 8305 neonates born in Jinjiang City Hospital between January 2016 and December 2018 for CHD. The positive screening results (positive POX or positive cardiac auscultation) were confirmed with echocardiography, while any false negative results were identified through follow-up and parent feedback. Sensitivity, specificity, positive/negative predictive values, Youden's index, and the area under the receiver operator characteristic curve (AUC) of the single use and combined use of the two methods (a POX test and auscultation) were calculated, and the results were compared.

Results: Among 8305 neonates, 22 cases were positive for POX alone, of which 6 cases were diagnosed by echocardiography; 83 cases were positive for cardiac auscultation alone, of which 47 cases were diagnosed by echocardiography; and 8 cases were positive for both methods, all of which were confirmed by echocardiography. Four more cases were confirmed during follow-up. Sensitivity, specificity, and the positive and negative predictive values of combined screening were $93.85 \%, 99.37 \%, 53.98 \%$ and $99.95 \%$, respectively, while Youden's index was 0.93 , and the AUC was 0.966 . Sixty-five cases of CHD were diagnosed, the total incidence being $7.82 \%$, and a ventricular septal defect was found to be the most common type.

Conclusion: The combination of POX test and cardiac auscultation as a screening method for neonatal CHD can reduce missed diagnoses and increase the detection rate of CHD in newborn infants.
\end{abstract}

Keywords: neonates, congenital heart disease, screening, percutaneous oxygen saturation test, cardiac auscultation

\section{Introduction}

Congenital heart disease (CHD) is a common heart disease, which can be caused by the abnormal development of the heart and blood vessels during embryonic development, or the failure of related channels to close after birth, and it ranks first among all birth defects. ${ }^{1}$ There are many types of CHD, the most common being ventricular septal defect, atrial septal defect, patent ductus arteriosus, pulmonary artery stenosis, coarctation of the aorta, tetralogy of Fallot, and complete transposition of the great arteries. At present, the internationally recognized prevalence of CHD in live births is $7-8 \%$.

In China, about 150,000 to 200,000 infants are born with CHD every year. ${ }^{2}$ Although mild CHD has little or no impact, without a timely response severe CHD
Correspondence: Yu-Lin Zhang Department of Pediatric, Jinjiang Municipal Hospital, No. 16 of Luoshan Section, Jinguang Road, Jinjiang, 362200 .

People's Republic of China

Tel +86 I5905080055

Email zhamgyl@2Icn.com 
will have a serious impact on a patient's life, and heart failure, pneumonia combined with acidosis, and even death can occur. The symptoms of CHD may not be typical, or the medical staff may not be very familiar with them or lack an understanding of them, so CHD is prone to missed diagnosis and misdiagnosis. It has been found that, for various reasons, about $20-30 \%$ of CHD neonate infants in grass-roots hospitals in China are not effectively diagnosed and treated, resulting in their death within one year of birth. ${ }^{3}$ Therefore, it is vital that CHD newborn infants are diagnosed as early as possible so that everything can be done to improve their survival rate.

In the past, for postnatal diagnosis of CHD, clinical evaluation and echocardiography were often used clinically. However, the cost of echocardiography screening is high, and the workload is huge, so at present its use in most primary medical institutions is not common. Therefore, our hope is that certain high-risk groups can be screened using simple forms of technology, and then those who show symptoms can be diagnosed with echocardiography. This would effectively avoid the waste of resources and enable the timely diagnosis and treatment of critical CHD patients.

The Children's Hospital of Fudan University in Shanghai is a leading domestic screening unit for CHD and uses percutaneous oxygen saturation (POX) combined with clinical evaluation methods, with some positive results. Corresponding indicators for early screening include heart murmurs, POX, a family history of CHD, shortness of breath, and certain deformities. Since then, screening index methods have been improved several times. Comparative analysis has revealed that, in the diagnosis of CHD, POX combined with cardiac auscultation has high sensitivity and is not prone to misdiagnosis. This finding offers a direction for the screening of CHD. ${ }^{4,5}$

In this study, 8305 eligible live births were selected for screening at a single center between January 2016 and December 2018. Using POX combined with cardiac auscultation, the investigators determined the incidence of CHD in the newborns and explored the feasibility and reliability of this program for screening for CHD. However, it needs to be clear that the screening results are only to be used as preliminary judgment indexes for infants with suspected CHD and are not intended to be the "gold standard" for the diagnosis of CHD. In other words, the final diagnosis of infants with suspected CHD still needs to be made with the use of echocardiography.

\section{Information and Methods}

\section{Subjects}

A total of 8305 neonates born in a single center between January 2016 and December 2018 were screened 24-72 hours after birth. Among them, there were 582 premature babies with a gestational age less than 37 weeks, accounting for $7 \%$ of the total. The screening methods were POX and cardiac auscultation, both of which are non-invasive examinations. All the parents of the screened subjects were informed of the role and purpose of this study and signed the informed consent form.

\section{Research Methods}

All the newborns who met the requirements were screened using POX and cardiac auscultation. The POX test was performed by two neonatologists, who had received standard training and were experienced in testing, and the detection instrument was a blood oxygen saturation detector specific for newborns (Masimo Corporation, model: RAD-5). One of the doctors carried out the test when the infant was in a quiet state, and the other doctor waited for more than three seconds for the value to stabilize before recording the results, thereby reducing the influence of error factors. Two other experienced doctors with special standard training carried out the cardiac auscultation, using the same stethoscope for all the subjects and with percutaneous oxygen saturation detection at the same time. One of the doctors carried out the examination, which lasted for a minimum of 15 seconds, while the baby was asleep or in a state of calm, and another doctor recorded the examination results in detail. If any indicator was found to be positive during the examination, the diagnosis was regarded as positive, and the newborn was sent for an echocardiogram, which was performed by an echocardiologist who did not know the previous screening results. Those who were diagnosed as needing immediate intervention were then transferred through the city's green channel to the Children's Hospital Affiliated to Fudan University in Shanghai for treatment as appropriate. Those who were diagnosed with CHD but did not need intervention for the time being were asked to return to the hospital for an echocardiography review three months after birth. All the newborn infants with a negative screening result, but who were reported to have symptoms such as cyanosis, shortness of breath, and feeding difficulties, required follow-up. This was done by telephone feedback from 
the parents at the age of six weeks. The occurrence of CHD during the recording period was recorded. If the diagnosis was positive, it was a false negative case during the initial screening process. In the screening process, the simple screening program of neonatal CHD in the Pediatric Hospital of Fudan University was in reference to. ${ }^{6}$

\section{Neonate CHD Screening Training}

There are 10 physicians in the neonatology department of Jinjiang Hospital, all of them have undergone unified professional training. Thereinto, the neonatal POX screening and cardiac auscultation were completed by uniformly trained neonatologists, and the echocardiography was completed by a team of highly qualified cardiovascular sonographers, who had undergone unified training, to ensure data quality. Thus, neonatologists and sonographers jointly completed the diagnosis of neonatal CHD. The above physicians participated in the National Pediatric Critical and Complex Congenital Heart Disease Diagnosis and Treatment Class and the National Pediatric Echocardiography Clinical Application Class hosted by the Cardiovascular Center of the Pediatric Hospital of Fudan University in batches.

\section{The Judgment of Positive Screening Indicators The Positive Standards for POX}

The POX positive standards were as follows (if one criterion was met, it was considered positive for CHD screening): the percutaneous oxygen saturation of the right hand and either foot was between $90 \%$ and $94 \%$, or the difference in this index between the right hand and either foot was $>3 \%$, and the result remained unchanged in a recheck four hours later under the same conditions; or the percutaneous blood oxygen saturation of the right hand or either foot was $<90 \%$.

\section{The Positive Standards for Cardiac Auscultation}

When performing cardiac auscultation on the neonates, it was important to keep them in a quiet state, avoid noise interference, and keep the skin in the chest and heart auscultation area clean and dry. A stethoscope was placed in the heart valve auscultation area, and auscultation was performed in sequence as follows: the mitral valve area, the pulmonary valve area, the aortic valve area, the second auscultation area of the aortic valve, the tricuspid valve area, and the first to fifth intercostal space on the left edge of the sternum.
The evaluation of the intensity of cardiac murmurs was based on the Levine classification method, where cardiac murmurs mainly refer to systolic murmurs. Those with a heart murmur auscultation of grade 2 and above were regarded as positive.

\section{The Echocardiography Procedure}

Echocardiography was carried out on the positively screened newborns between the determination of a positive result and one week of birth. The GEVIVIDE9 probe model (S8-3, 4-8 Hz) was used to carry out the echocardiography. The neonates were examined by ultrasound whilst in a quiet state. The echocardiogram examined the four heart chambers and the suprasternal fossa section. If CHD was diagnosed, auxiliary detection was carried out in other sections. The echocardiographic recordings of the subjects diagnosed with CHD were saved in AVI mode, and experienced doctors from the cardiovascular ultrasound department were called for a reexamination, which had to take place within 24 hours to ensure the quality of the screening. All imaging data were kept for future reference.

\section{Diagnostic Criteria for CHD}

A diagnosis of CHD was based on echocardiography as well as the relevant specifications of the international pediatric and CHD code, ${ }^{7}$ which take into account the coexistence of various cardiac malformations. The malformations that have the greatest impact on hemodynamics or need to be treated first were included in the statistics, but secondary deformities were not counted. The CHD exclusion criteria were as follows: children with patent ductus arteriosus were re-examined by echocardiography three months after birth, and if the arterial duct could be closed, they were not included in the statistics, and patients with a septal defect $<5 \mathrm{~mm}$, a patent foramen ovale, or mild to moderate tricuspid valve regurgitation were also not included in the statistics. $^{8}$

\section{Statistical Analysis Statistical Methods}

Data arrangement and statistical analysis were conducted using statistical software SPSS24.0. The intergroup comparison of counting data was carried out using a Chisquare test and expressed as a percentage (\%). A p-value $<0.05$ was considered statistically significant. 


\section{Results \\ CHD Detection Results}

Between January 2016 and December 2018, 8425 live neonates were delivered in one single center obstetrics department, but 120 of them were transferred to the neonatal intensive care unit or another hospital within 24 hours of birth, so they were not included in the CHD screening. Thus, a total of 8305 neonates were included in the CHD screening, amounting to a screening rate of 98.5\%. As a result of the POX and cardiac auscultation screening, 22 cases $(0.26 \%)$ were found positive for POX alone, 6 cases of which were then diagnosed as having CHD after echocardiography, so the diagnosis rate was $27.2 \%$ in the POX group; 83 cases were positive for cardiac auscultation alone, accounting for $0.99 \%$ of the total 8305 screened cases, of which 47 cases were diagnosed after echocardiography, making a $56.6 \%$ diagnosis rate for the cardiac auscultation group; and 8 cases were positive for both examinations, accounting for $0.09 \%$ of the total screened cases, all of whom were diagnosed with CHD after echocardiography, meaning the diagnosis rate was $100 \%$ in the double positive group.

A false negative was found in 4 cases in the follow-up. They were all negative for POX and cardiac auscultation at 24-72 hours after birth, but by the age of six weeks, through telephone follow-up and parents' feedback, it was found that they had developed symptoms of CHD, such as cyanosis, ecphysesis and feeding difficulties. A diagnosis of CHD was then confirmed with echocardiography.

In addition, during the initial screening process, among the 22 neonates positive for POX alone, 6 of them were diagnosed with CHD after echocardiography, and 16 were excluded. Of the 6 confirmed infants, 3 of them had transposition of the great arteries, 2 of them had anomalous pulmonary venous drainage, and 1 of them had pulmonary atresia. Among the 16 false-positive POX cases, 6 of them were diagnosed with persistent pulmonary arterial hypertension, and 10 of them were diagnosed with neonatal pneumonia. Among the 83 neonates positive for cardiac auscultation alone, 47 of them were diagnosed with CHD after echocardiography, and 36 of them were excluded. Of these 47 confirmed infants, 26 of them had a ventricular septal defect, 10 of them had an atrial septal defect $(>5 \mathrm{~mm}), 4$ of them had patent ductus arteriosus, 4 of them had pulmonary valve stenosis, and 3 of them had aortic coarctation. Of these 36 cases with false-positive cardiac auscultation, echocardiography found no abnormality in 13 of them, while in 23 neonates echocardiography revealed a patent foramen ovale, atrial septal defect $(<5 \mathrm{~mm})$, patent ductus arteriosus. These cases were expected to improve as the child grew, so they were considered normal. The study found through follow-up that echocardiogram showed a total of 2 children with patent ductus arteriosus returned to normal after 1 month of birth, and the other 2 children with patent ductus arteriosus recovered after 2 months of birth, and there was no duct fistula. All of the 8 infants found positive for both POX and cardiac auscultation were diagnosed with $\mathrm{CHD}$ after echocardiography, and 3 of them had tetralogy of Fallot, 1 of them had ventricular septal defect, 2 of them had patent ductus arteriosus, 1 of them had pulmonary valve stenosis, and 1 of them had double-outlet right ventricle (Figure 1, Table 1).

\section{Types and Composition of Neonatal $\mathrm{CHD}$}

Sixty-one of the positively screened infants were diagnosed with CHD using color Doppler ultrasound, and 4 false negative cases were found in the follow-up, so a total of 65 cases of CHD were diagnosed. A total of 65 cases were diagnosed not only in neonatal period but within 6 weeks of age. When the types of defect were analyzed statistically, the results revealed that ventricular septal defect, atrial septal defect, and patent ductus arteriosus (including other cardiac malformations) were the top three, accounting for $41.5 \%, 18.4 \%$, and $12.3 \%$ respectively. Eighteen cases had complex CHD (3 cases had transposition of the great arteries, 1 case had double-outlet right ventricle, 5 cases had pulmonary valve stenosis, 3 cases had tetralogy of Fallot, 3 cases had aortic coarctation, 2 cases had anomalous pulmonary venous drainage, and 1 case had pulmonary atresia). Of the 4 false negative cases, 2 had atrial septal defect $(>5 \mathrm{~mm}$ ), while the other 2 had patent ductus arteriosus (which was not closed at three months after birth) (Table 2).

\section{The Detection of 65 Cases of Neonatal CHD Using the Different Screening Methods}

Table 3 shows the sensitivity of different screening methods for patients with CHD. The results revealed that pulse blood oxygen saturation combined with cardiac murmur detection has the highest detection rate. Compared with the single cardiac murmur test, pulse blood oxygen saturation measurement has a higher 


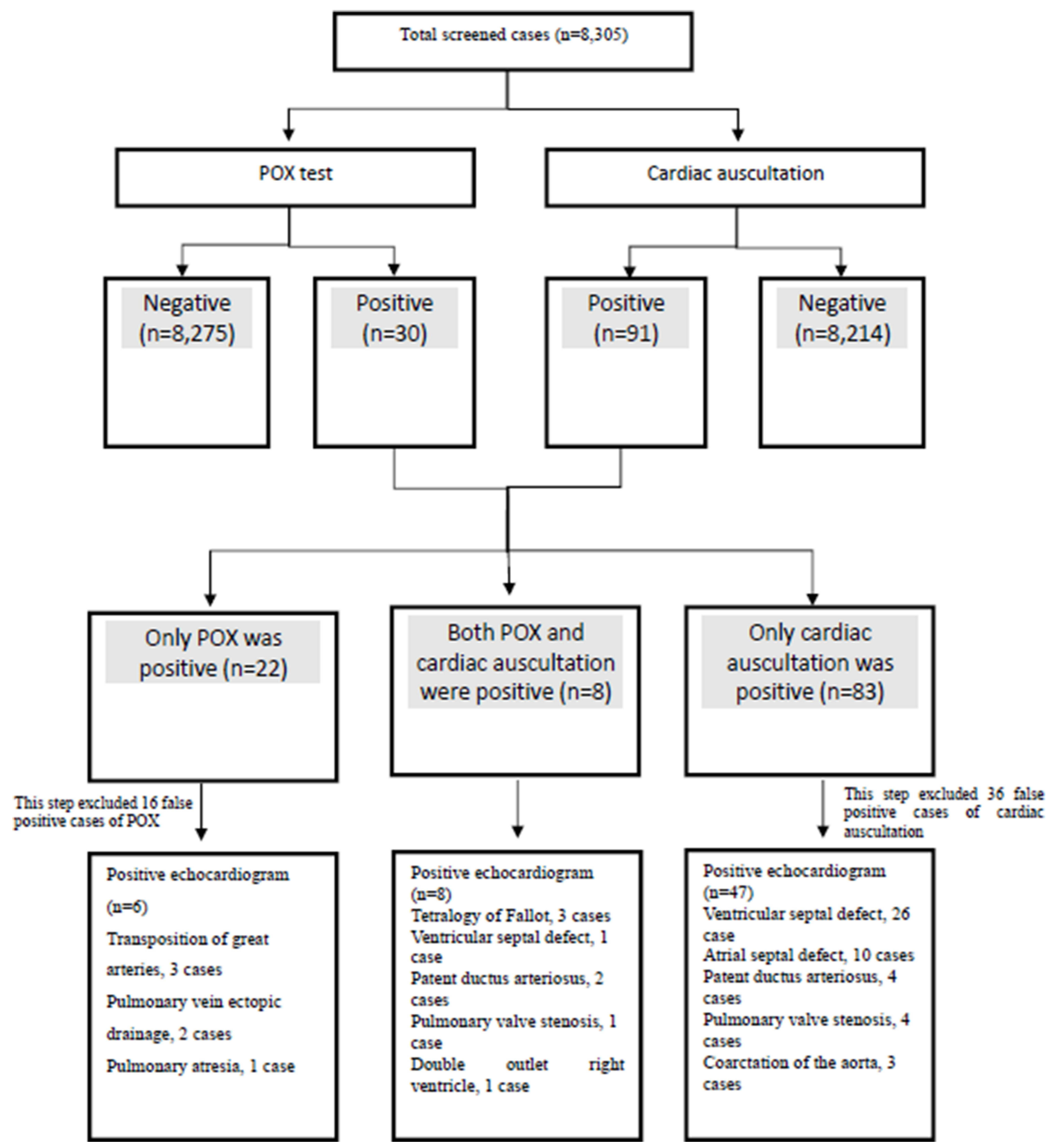

Figure I POX and cardiac auscultation double-blind screening process.

detection rate for complete transposition of great arteries, anomalous pulmonary venous drainage, and pulmonary atresia, while cardiac auscultation examination is more likely to find atrial septal defect, ventricular septal defect, patent ductus arteriosus, pulmonary valve stenosis, and aortic coarctation.

\section{A Comparison of the Sensitivity,} Specificity, and Youden's Index of Three Screening Methods for Neonatal CHD

A total of 8305 infants were screened and followed up after discharge, and 65 cases of CHD were found using echocardiography, which was regarded as the "gold standard." The results 
Table I Positive Detection of CHD Screening Indicators

\begin{tabular}{|l|c|c|c|c|}
\hline Screening Indicators & $\begin{array}{c}\text { Positive } \\
\text { Cases(n) }\end{array}$ & $\begin{array}{c}\text { Positive } \\
\text { Percentage(\%) }\end{array}$ & $\begin{array}{c}\text { Diagnosed Cases by Color Doppler } \\
\text { Ultrasound(n) }\end{array}$ & $\begin{array}{c}\text { Positive Rate of } \\
\text { Diagnosis (\%) }\end{array}$ \\
\hline POX positive alone & 22 & 26.0 & 6 & 27.2 \\
Positive cardiac & 83 & 99.0 & 47 & 56.6 \\
auscultation alone & 8 & 9.0 & 8 & 100 \\
Double positive & 8 & & \\
\hline
\end{tabular}

Table 2 Various Types of CHD

\begin{tabular}{|l|c|c|c|c|}
\hline CHD Type & $\mathbf{n}$ & False Negative (n) & Composition Ratio (\%) & Incidence (\%) \\
\hline Ventricular septal defect & 27 & 41.5 & 3.25 & 18.4 \\
Atrial septal defect & 10 & +2 & 12.3 & 0.61 \\
Patent ductus arteriosus & 6 & +2 & 0.36 \\
Pulmonary valve stenosis & 5 & 7.6 & 0.36 \\
Tetralogy of Fallot & 3 & 4.6 & 0.36 \\
Transposition of great arteries & 3 & 4.6 & 0.24 \\
Coarctation of the aorta & 3 & 4.6 & 0.12 & 0.12 \\
Anomalous pulmonary venous drainage & 2 & 3.0 & 100 \\
Pulmonary atresia & 1 & 0.12 & 0.12 & \\
Double outlet right ventricle & 1 & +4 & 7.82 \\
Total & 61 & 0.96 & \\
\hline
\end{tabular}

Notes: Most patients have only one type of deformity, and a small number of them have multiple types of deformities, for statistical convenience, only one major deformity was included. Major deformities refer to the deformities that have the greatest impact on hemodynamics or require primary treatment, and no statistics on minor deformities, for example, in case of VSD combined with small ASD, VSD will be counted, and small ASD will not be included in the statistics, in case of TGA combined with VSD, TGA will be counted, and VSD will no longer be included in the statistics.

Table 3 Comparison of Detection Rates of POX, Cardiac Auscultation, and Combined Screening

\begin{tabular}{|c|c|c|c|c|}
\hline Disease Type & Number of Confirmed Cases & $\begin{array}{l}\text { POX } \\
n(\%)\end{array}$ & $\begin{array}{c}\text { Cardiac Auscultation } \\
\mathbf{n}(\%)\end{array}$ & $\begin{array}{c}\text { Combined Screening } \\
\mathbf{n}(\%)\end{array}$ \\
\hline Transposition of great arteries & 3 & $3(100)$ & 0 & $3(100)$ \\
\hline Anomalous pulmonary venous drainage & 2 & $2(100)$ & 0 & $2(100)$ \\
\hline Pulmonary atresia & 0 & $\mathrm{I}(100)$ & 0 & $\mathrm{I}(100)$ \\
\hline Tetralogy of Fallot & 3 & $3(100)$ & $3(100)$ & $3(100)$ \\
\hline Ventricular septal defec & 27 & $I(37.0)$ & $27(100)$ & $27(100)$ \\
\hline Patent ductus arteriosus & 8 & $2(25.0)$ & $6(75.0)$ & $6(75.0)$ \\
\hline Pulmonary valve stenosis & 5 & $\mathrm{I}(20.0)$ & $5(100)$ & $5(100)$ \\
\hline Double outlet right ventricle & 1 & $\mathrm{I}(100)$ & $\mathrm{I}(100)$ & $I(100)$ \\
\hline Atrial septal defect & 12 & 0 & $10(83.3)$ & $10(83.3)$ \\
\hline Coarctation of the aorta & 3 & 0 & $3(100)$ & $3(100)$ \\
\hline
\end{tabular}

also showed that the sensitivity of POX combined with cardiac auscultation was $93.85 \%$, indicating that the dual method can significantly improve screening sensitivity and Youden's index, while the figures for effectiveness and authenticity were also the highest (Table 4).

\section{A Comparison of Receiver Operating Characteristic Curve of Subjects Using the Different Screening Methods}

The receiver operating characteristic (ROC) curves of the three screening methods were compared and analyzed, and 
Table 4 Comparison of Sensitivity, Specificity and Youden's Index of 3 Methods (Cases)

\begin{tabular}{|l|c|c|c|}
\hline Methods & POX & Cardiac Auscultation & Combined Screened \\
\hline True negative (case) & 14 & 55 & 61 \\
False negative (case) & 16 & 36 & 52 \\
Sensitivity (\%) & 8224 & 8204 & 8188 \\
Specificity (\%) & 51 & 10 & 4 \\
Positive predictive value (\%) & $21.54(14 / 65)$ & $84.62(55 / 65)^{*}$ & $93.85(61 / 65)^{\#+}$ \\
Negative predictive value (\%) & $99.80(8224 / 8240)$ & $99.56(8204 / 8240)^{*}$ & $99.37(8188 / 8240)^{\#+}$ \\
Youden's index & $46.67(14 / 30)$ & $60.44(55 / 91)^{*}$ & $53.98(61 / 113)^{\#+}$ \\
True negative (case) & $99.38(8224 / 8275)$ & $99.88(8204 / 8214)^{*}$ & $99.95(8188 / 8192)^{\#+}$ \\
False negative (case) & 0.21 & 0.84 & 0.93 \\
\hline
\end{tabular}

Notes: *Comparison between cardiac auscultation and POX, $\mathrm{P}<0.05 ;{ }^{*}$ Comparison between combined screened and POX, P<0.05; ${ }^{+}$Comparison between combined screened and cardiac auscultation, $\mathrm{P}<0.05$; Youden's index: Youden's index is the sum of sensitivity and specificity minus I, it reflects the judgment performance of screening methods on patients and no patients, and there is a positive correlation between the value and the screening effect, the larger the value is, the greater the authenticity is.

the results showed that the area under the ROC curve (AUC) of combined screening was 0.966 , which was significantly higher than the AUC of a single method screening, and the ability for the combined screening was also the highest (Figure 2, Table 5).

\section{Discussion}

\section{Neonatal Screening and the Early Diagnosis of CHD}

At present, the internationally recognized incidence rate of CHD is $7 \%{ }^{-}-8 \%$. In the present study, 65 children were diagnosed with CHD making the incidence of CHD in our city $7.82 \%$. ${ }^{9}$ Among 120 referrals not included by screening, 22 of them had a prenatal diagnosis of severe CHD, which is why they were transferred to other hospitals within six hours of birth. Fifteen of them had complex CHD, 5 of them had atrial septal defect, and 2 of them had ventricular septal defect. In addition, most of the infants transferred for other reasons had an extremely low birth weight and congenital malformations. Since the incidence of CHD in these infants is often higher than that in other infants in hospital, ${ }^{10}$ timely tracking these infants' CHD screening results in other hospitals can better reflect the detection rate of CHD screening in newborns.

In addition, in this study, the false negative cases were found at the 6-week-old follow-up. There were 4 cases in total, 2 of which had atrial septal defect ( $>5 \mathrm{~mm}$ ), and 2 of which had patent ductus arteriosus (which was not closed three months after birth). According to the follow-up results of the Pediatric Hospital Affiliated to Shanghai Fudan University, the rate of missed diagnosis was $3.40 \%$, whereas in this study, the rate of missed diagnosis was $6.15 \%$. The team from the Pediatric Hospital Affiliated to Shanghai Fudan University conducted a follow-up study on 27,201 neonates with initial negative screening results and normal follow-up results at 6 weeks old and found that the probability of diagnosis of CHD due to symptoms was $0.1 \%$ Therefore, it would appear that such newborns are less likely to be false negative cases.

\section{The Effectiveness of POX Combined with Cardiac Auscultation as a Screening Method for Neonatal CHD}

Among the 6 neonates diagnosed by POX screening alone, 1 with anomalous pulmonary venous drainage was found to have cyanosis six hours after birth, and it was recorded that the difference in transcutaneous oxygen saturation between the right hand and either foot was $>3 \%$; the other 5 neonates were cyanotic from birth. Therefore, in neonates with transposition of the great arteries, anomalous pulmonary venous drainage, and pulmonary atresia, although postnatal murmurs may not be obvious, the POX can be abnormal. Thus, a postnatal blood oxygen saturation test would appear to play a guiding clinical role in the early detection of such CHD infants. Among the 16 false positive POX cases, 6 cases were diagnosed with persistent pulmonary arterial hypertension, and 10 cases were diagnosed with neonatal pneumonia. The interference of factors such as poor peripheral circulation in neonatal hypothermia was excluded from being the reason for the false positivity. During the examination, hypoxemia caused by pulmonary factors was the most prevalent. Among the false negative POX cases, the results of this study revealed that most infants with CHD had no right-to-left shunt of blood in the left and right sides 


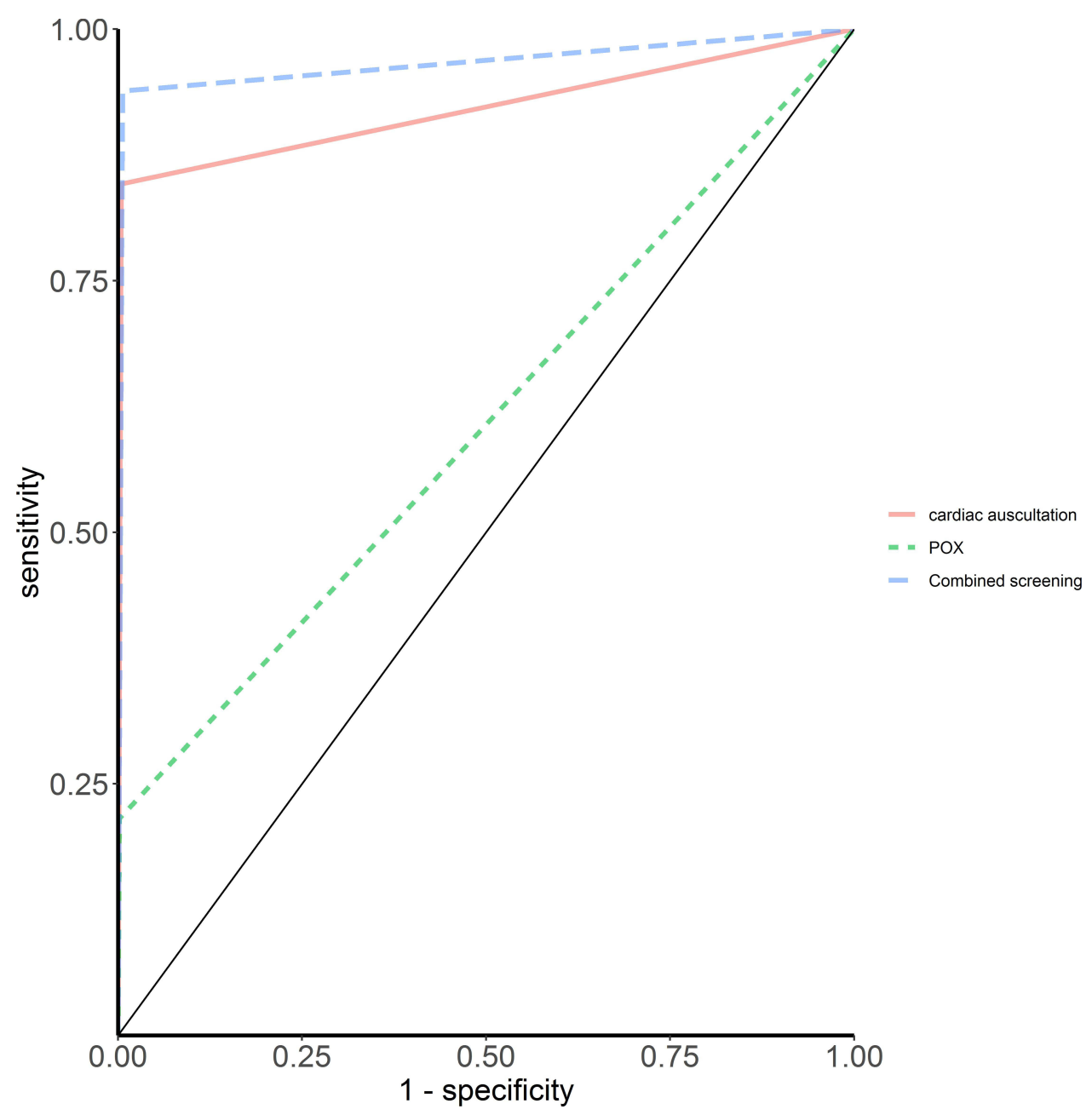

Figure 2 Comparison of ROC curves among different screening methods.

of the heart, so the corresponding blood oxygen saturation did not change significantly. However, when a heart defect is mild, the pulmonary artery pressure is normal and, in a quiet state, a blood oxygen saturation test may also be negative. The results of this study showed that the accuracy, sensitivity, and specificity of POX were $99.19 \%, 21.54 \%$, and $99.80 \%$, respectively, suggesting that the sensitivity of a POX test is not high, and the possibility of missed

Table 5 Comparison of ROC Curves of Subjects with Different Screening Methods

\begin{tabular}{|l|l|l|l|}
\hline Diagnostic Method & AUC & SEM & 95 \% Cl \\
\hline Blood oxygen saturation & 0.607 & 0.041 & $0.527 \sim 0.687$ \\
Heart murmur & 0.921 & 0.026 & $0.869 \sim 0.973$ \\
Combined screening & 0.966 & 0.017 & $0.932 \sim 1.000$ \\
\hline
\end{tabular}

Note: The differences in the AUC among 3 curves were statistically significant $(P<0.001)$. diagnosis is high in some newborn infants with CHD who have obvious hypoxemia. The results indicate that POX alone has limitations in screening for CHD, and, therefore, it cannot completely replace clinical evaluation. This is consistent with the results of related studies conducted at Leipzig University in Germany, between 2006 and 2008, and relayed studies conducted by Thangaratinam et al in 2012. ${ }^{11,12}$

Cardiac auscultation is another method that is widely used in clinical screening for CHD. In the present study, most of the newborn infants were found to have CHD because of positive cardiac auscultation. Therefore, cardiac auscultation is of great significance in screening for CHD. However, the type of CHD makes a difference, and the loudness of murmur is not directly proportional to the degree of heart deformity. For example, complete transposition of the great arteries does not cause cardiac murmur or, at least, it does not cause any obvious cardiac 
murmur in the early postnatal period. Research has shown that about $55 \%$ of infants with CHD have no heart murmur, and so $82 \%$ of infants are not diagnosed at discharge because of a normal clinical evaluation result, ${ }^{13}$ suggesting that murmur is not a specific index for screening for CHD. In this study, there were 10 false negative cases of cardiac auscultation alone. The reasons may be as follows: (1) with respect to anatomical location, ventricular function and other related factors, although some neonates do not present with murmur, echocardiography will yield a diagnosis of CHD; (2) the hospital environment has an impact, so although some infants have transient cardiac murmur, it may not be heard because of the surrounding noise during auscultation; and (3) the professional skills of the doctors will also have a significant effect on the test results. Among the 36 cases of false positive cardiac auscultation, no abnormality was found in 13 cases during echocardiography, while in 23 cases echocardiography showed a patent foramen ovale, atrial septal defect $(<5 \mathrm{~mm})$, patent ductus arteriosus. Since these cases were expected to improve over time, they were considered normal. Therefore, there is a definite possibility of a missed diagnosis when using this index alone to diagnose CHD because even if no cardiac murmur is detected, CHD may exist. On the other hand, there may be no CHD in the case of a positive heart murmur in auscultation. Such a misdiagnosis increases the psychological and economic burden on family members. In this study, 65 of the screened newborn infants were diagnosed with CHD, 55 of whom were finally diagnosed with CHD because of positive heart murmur in auscultation screening. Thus, the sensitivity of heart murmur in CHD diagnosis was $84.62 \%$. The foregoing discussion suggests that using POX or cardiac auscultation alone has limitations in accurate screening for neonatal $\mathrm{CHD}$, and, therefore, it is necessary to combine the two. This view has become the key research direction in this field.

In this study, the accuracy, sensitivity, specificity, positive/negative predictive value, Youden's index, and the AUC of the single use or combined use of the two methods were calculated, and the results were compared. The combined screening method had high sensitivity (93.85\%) as did Youden's index, and the AUC was 0.966. These results were all higher than those of POX or cardiac auscultation alone, and this combination exhibited strong screening ability, allowing for the identification of potential CHD infants in the early postnatal period. Both POX and cardiac auscultation are simple, easy to operate, noninvasive, and low cost, and their combined use can greatly reduce the proportion of missed diagnosis and misdiagnosis and the proportion of other unnecessary clinical examinations, thereby saving valuable medical resources.

\section{Types of CHD}

Ventricular septal defect accounts for $41.5 \%$ of all CHDs, making it the most common type of CHD in the neonatal period, and the incidence of atrial septal defect and patent ductus arteriosus rank second and third. ${ }^{14,15}$

Although the majority of CHD is a single malformation, such as atrial septal defect or pulmonary artery stenosis, there are also a few complex malformations, such as transposition of the great arteries, which are arterial duct dependent. Proper prenatal fetal echocardiography can reduce the incidence of complex CHD to a certain extent, however, and this study screened out 3 cases of transposition of the great arteries, 1 case of double outlet right ventricle, 5 cases of pulmonary valve stenosis, 3 cases of tetralogy of Fallot, 3 cases of aortic coarctation, 2 cases of anomalous pulmonary venous drainage, and 1 case of pulmonary atresia, amounting to a total incidence of $2.17 \%$. Therefore, POX combined with cardiac auscultation screening is also of great significance in the early and accurate diagnosis of complex CHD.

The data in Table 4 show that pulse blood oxygen saturation combined with cardiac murmur detection has the highest detection rate $(100 \%)$. The combination of POX and cardiac auscultation to screen neonates can improve the accuracy and sensitivity of screening for CHD, reduce the rate of missed diagnosis and misdiagnosis, and improve the reliability of screening results.

However, in this study, only the positively screened patients underwent echocardiography, and there were missed diagnoses. For example, the auscultation of neonatal cardiac murmur is usually negative in patients with small atrial septal defects or whose murmurs are not obvious, and oxygen saturation may also be normal. Such missed diagnoses may not have a great impact, but for others a missed diagnosis may have adverse consequences. Patients with abnormal arteries, such as a coarctation of the aortic arch, which is not serious at birth, or a pulmonary arteriovenous fistula, may have negative results for both POX and cardiac auscultation. Therefore, medical staff should increase the clinical indicators according to the actual situation to reduce 
the missed diagnosis rate of screening, and patients with negative screening should be followed up so that any problems can be found in time. In this way, primary hospitals in China can more effectively carry out CHD screening and determine the screening process according to the actual situation. Generally, doctors in primary hospitals are proficient in POX and cardiac auscultation, and the procedures are not difficult. Therefore, it is feasible to promote this combined screening method at the grass-roots level, particularly due to its high application value.

\section{Limitations}

There were also some limitations to the screening of CHD in this study; most importantly, four false negative infants were found. Therefore, improvements are necessary. Firstly, the accuracy of cardiac auscultation should be improved with regular staff training, so that newborn infants with heart murmur can be found in time and the rate of missed diagnosis reduced. Secondly, at a later stage, through follow-up and feedback from parents, echocardiography should be carried out on children suspected of having CHD. Although this will inevitably lead to the missing of some false negative cases, for example, infants with mild heart disease who often have normal cardiac auscultation and POX test results, in the case of a large sample size, missing diagnoses are inevitable, so strengthening health education and follow-up after discharge is the only practical way of keeping diagnostic errors to a minimum.

\section{Ethics Approval and Consent to Participate}

This study was conducted with approval from the Ethics Committee of Jinjiang municipal hospital. This study was conducted in accordance with the declaration of Helsinki. Written informed consent was obtained from all participants.

\section{Disclosure}

The authors report no conflicts of interest in this work.

\section{References}

1. Duess JW, Puri P. Syndromic hirschsprung's disease and associated conge-nital he-art disease: a systematic review. Pediatr Surg Int. 2015;31(8):781-785. doi:10.1007/s00383-015-3744-6

2. Cawsey MJ, Noble S, Crosssudworth F, et al. Feasibility of pulse oximetry screening for critical congenital heart defects in homebirths. Arch Dis Child Fetal Neonatal Ed. 2016;101(4):349-351. doi:10.1136/archdischild-2015-309936

3. Krishnamurthy G, Ratner V, Bacha E, et al. Comorbid conditions in neo-nates with congenital heart disease. Pediatr Care Med. 2016;17 (8 Suppl 1):S367-376. doi:10.1097/PCC.0000000000000832

4. Zhao Q, Liu F, Wu L, et al. Analysis of missed diagnosis in neonatal obstetric hospital with critical congenital heart disease before discharge. Int J Pediatr. 2017;68(4):260-266. (in Chinese with English abstract).

5. Wong KK, Fournier A, Fruitman DS, et al. Canadian cardiovascular society cana-dian pediatric cardiology association position statement on pulse oximetry screening in newborns to enhance detection of critical congenital heart disease. Can J Cardiol. 2017;33(2):199-208. doi:10.1016/j.cjca.2016.10.006

6. Hu X, Huang G. Research progress of neonatal congenital heart disease screening. Int J Pediatr. 2014;411(2):120-123. (in Chinese with English abstract).

7. Simeone RM, Tinker SC, Gilboa SM, et al. Proportion of selected congenital heart defects attributable to recognized risk factors. Ann Epidemiol. 2016;26(12):838-845. doi:10.1016/j. annepidem.2016.10.003

8. Chantepie A, Soulé N, Poinsot J, et al. Heart murmurs in asymptomatic children: when should you refer? Arch Pediatr. 2016;23 (1):97-104. doi:10.1016/j.arcped.2015.10.006

9. Bruno CJ, Havranek T. Screening for critical congenital heart disease in newborns. Adv Pediatr. 2015;62(1):211-226. doi:10.1016/j. yapd.2015.04.002

10. Bestetti RB, Dellalibera-Joviliano R, Lopes GS, et al. Determination of the Th1, Th2, Th17, and Treg cytokine profile in patients with chronic chagas heart disease and systemic arterial hypertension. Heart and Vessels. 2019;34(1):123-133. doi:10.1007/s00380-018$1228-\mathrm{z}$

11. Thangaratinam S, Brown K, Zamora J, et al. Pulse oximetry screening for critical congenital heart defects in asymptomatic newborn babies:a sys-tematicreviewand meta analysis. Lancet. 2012;379 (9835):2459-2464. doi:10.1016/S0140-6736(12)60107-X

12. Stoll C, Dott B, Alembik Y, et al. Associated noncardiac congenital anomalies among cases with congenital heart defects. Eur $J$ Med Genet. 2015;58(2):75-85. doi:10.1016/j.ejmg.2014.12.002

13. Riede FT, Wörner C, Dähnert I, Möckel A, Kostelka M, Schneider P. Effectiveness of neonatal pulse oximetry screening for detection of critical congenital heart disease in daily clinical routine--results from a prospective multicenter study. Eur J Pediatr. 2010;169(8):975-981. doi:10.1007/s00431-010-1160-4

14. Olney RS, Ailes EC, Sontag MK. Detection of critical congenital heart defects: review of contributions from prenatal and newborn screening. Semin Perinatol. 2015;39(3):230-237. doi:10.1053/j. semperi.2015.03.007

15. Powell R, Pattison HM, Bhoyar A, et al. Pulse oximetry as a screening test for congenital heart defects in newborn infants:an evaluation of acceptability to mothers. Arch Dis Child. 2013;98:F59F63. doi:10.1136/fetalneonatal-2011-301225 


\section{Publish your work in this journal}

The International Journal of General Medicine is an international, peer-reviewed open-access journal that focuses on general and internal medicine, pathogenesis, epidemiology, diagnosis, monitoring and treatment protocols. The journal is characterized by the rapid reporting of reviews, original research and clinical studies across all disease areas. The manuscript management system is completely online and includes a very quick and fair peer-review system, which is all easy to use. Visit http://www.dovepress.com/ testimonials.php to read real quotes from published authors.

Submit your manuscript here: https://www.dovepress.com/international-journal-of-general-medicine-journal 